\title{
Immunization registries in the EMR Era
}

\author{
Lindsay A. Stevens ${ }^{1,2}$, Jonathan P. Palma ${ }^{1,2}$, Kiran K. Pandher ${ }^{3}$, Christopher A. Longhurst ${ }^{1,2}$
}

1 Department of Pediatrics, Stanford University School of Medicine, Stanford, California, 2 Department of Clinical Informatics, Lucile Packard Children's Hospital, Palo Alto, California, 3 Department of Information Services, Lucile Packard Children's Hospital, Menlo Park, California

\section{Abstract}

Background: The CDC established a national objective to create population-based tracking of immunizations through regional and statewide registries nearly 2 decades ago, and these registries have increased coverage rates and reduced duplicate immunizations. With increased adoption of commercial electronic medical records (EMR), some institutions have used unidirectional links to send immunization data to designated registries. However, access to these registries within a vendor EMR has not been previously reported.

Purpose: To develop a visually integrated interface between an EMR and a statewide immunization registry at a previously non-reporting hospital, and to assess subsequent changes in provider use and satisfaction.

Methods: A group of healthcare providers were surveyed before and after implementation of the new interface. The surveys addressed access of the California Immunization Registry (CAIR), and satisfaction with the availability of immunization information. Information Technology (IT) teams developed a "smart-link" within the electronic patient chart that provides a single-click interface for visual integration of data within the CAIR database.

Results: Use of the tool has increased in the months since its initiation, and over 20,000 new immunizations have been exported successfully to CAIR since the hospital began sharing data with the registry. Survey data suggest that providers find this tool improves workflow and overall satisfaction with availability of immunization data. $(p=0.009)$.

Conclusions: Visual integration of external registries into a vendor EMR system is feasible and improves provider satisfaction and registry reporting.

Key Words: Electronic Medical Records, Immunization Registries, EMR integration, HITECH, Meaningful Use

Abbreviations: California Immunization Registry (CAIR), Computerized Physician Order Entry (CPOE), Electronic Medical Record (EMR), Health Information Technology for Economic and Clinical Health Act (HITECH), Health Level 7 (HL7), Immunization Information System (IIS), Information Technology (IT), Lucile Packard Children's Hospital (LPCH) 
Correspondence: lindsay.stevens@stanford.edu

Copyright @2013 the author(s)

This is an Open Access article. Authors own copyright of their articles appearing in the Online Journal of Public Health Informatics. Readers may copy articles without permission of the copyright owner(s), as long as the author and OJPHI are acknowledged in the copy and the copy is used for educational, not-for-profit purposes.

\section{Introduction}

Since their inception nearly 20 years ago, immunization registries have been shown to increase vaccine coverage rates and decrease duplicate immunizations. ${ }^{1-3}$ The CDC provides funding for immunization information system (IIS) programs in all 50 states. ${ }^{4}$ As part of the Health Information Technology for Economic and Clinical Health Act (HITECH) of 2009, providers with electronic medical records (EMRs) are encouraged to submit electronic immunization data to IISs to achieve meaningful use. ${ }^{4-6}$ Many institutions have established unidirectional interoperability by which their EMR immunization records are uploaded to a designated registry - thereby preventing the need for staff to manually update each patient chart in the registry. ${ }^{5-1}$ Bidirectional interoperability is preferable as it would allow accurate immunization data from the IIS to be sent directly to the hospital's or practice's EMR, however integration is expensive and difficult to implement. ${ }^{11}$ Visual integration into the EMR with context-sensitive access can meet clinician needs without the expense of bidirectional data integration.

Lucile Packard Children's Hospital (LPCH) has a strong history of developing informaticsenabled innovations. ${ }^{12-16}$ LPCH had not, however, previously participated in the statewide immunization registry, the California Immunization Registry (CAIR), largely due to concerns for duplication of efforts by clinical staff who would need to manually enter the same data in both the vendor EMR and the IIS. The previous system was paper-based and involved scanning copies of the patient records in to the EMR. No immunization information was tracked directly in our EMR. This information was not complete and only contained data for those immunizations recorded at $\mathrm{LPCH}$.

The few published reports on bidirectional registries involved Health Level 7 (HL7) web service, which have not been widely replicated given that the technical work involved is far greater and difficult to sustain. ${ }^{17,18}$ Decision support services in the EMR are much more limited as the compared to those native in the registries. Our team's goal was to develop a visually integrated interface by which clinical staff could quickly and easily access the registry to evaluate a patient's immunization history from within our EMR. It was hypothesized that initiating the association between LPCH and CAIR would add to the number of patients included in the IIS, increase provider utilization of this tool, and improve provider satisfaction and perceived efficiency. 


\section{Methods}

\section{Integration}

Creation of the visually integrated registry interface within the vendor EMR required two different efforts. The first was to upload hospital immunization data to the IIS, transferring nightly any new immunizations recorded at LPCH using HL7 code. Historical data, including all immunizations previously ordered using Computerized Physician Order Entry (CPOE) from, were also uploaded. The second aspect was to create a "smart link" - a web-based icon in the "Patient Summary" area of the chart that directs providers to the CAIR registry site. (Figure 1). Clicking this link sends patient identifiers to the CAIR database, using an institutional login to access the patient's CAIR chart. The CAIR interface includes both immunizations from LPCH data uploads and any others added to the database by outside institutions, as well as its native decision support tool. (Figure 2). The new interface was implemented at the end of the 2011 calendar year.

\section{Patient Summary}

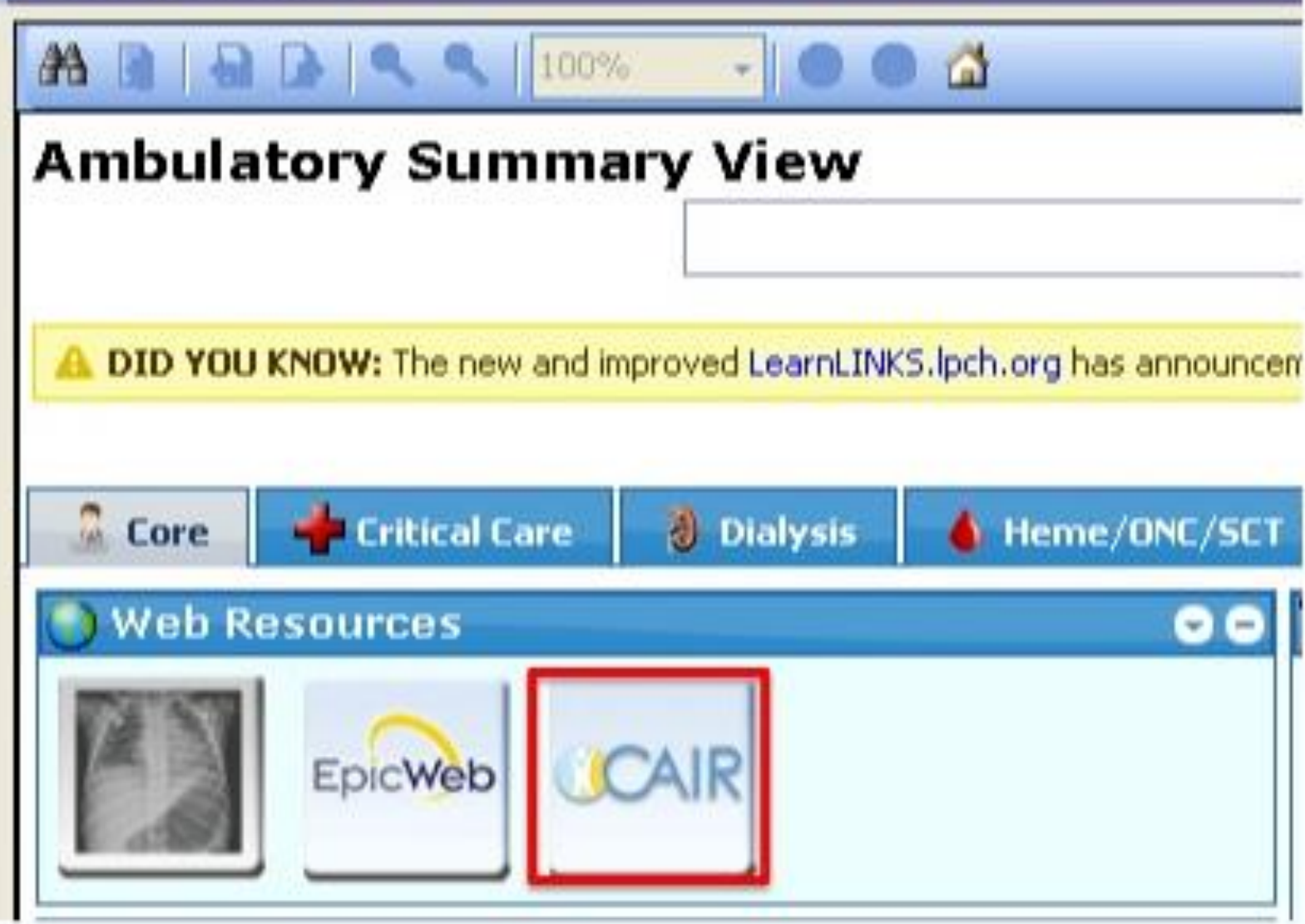

Figure 1: Screen shot of CAIR link in patient chart 


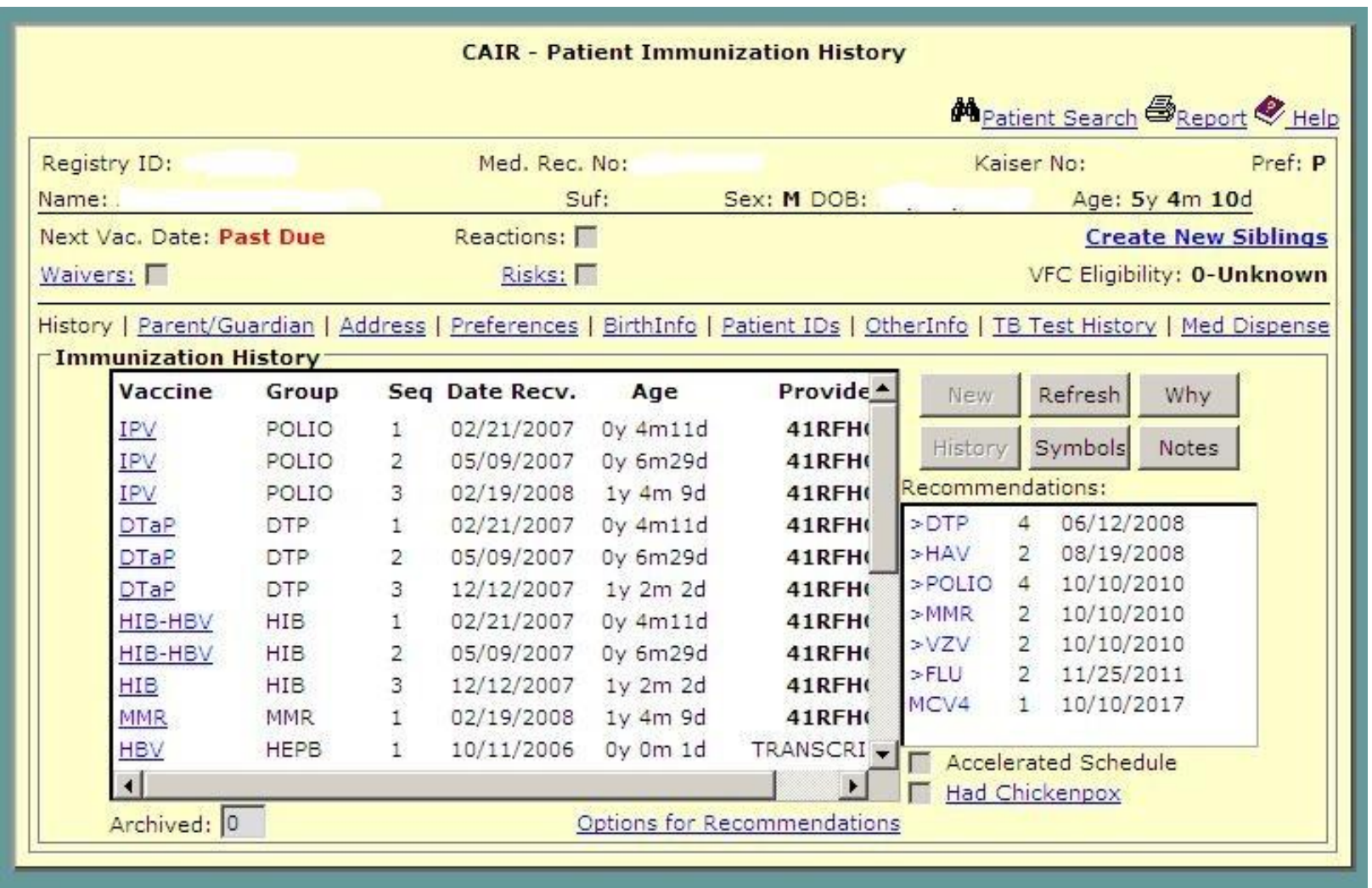

Figure 2: Screen shot of the patient's immunization record that is accessed when the link is clicked.

\section{Survey}

Healthcare providers at LPCH were electronically surveyed several months prior to the rollout of the tool with regards to their satisfaction with the prior paper system and their use of the statewide registry $(n=41)$. All LPCH residents, General Pediatrics attending physicians, primary care clinic nurses and nursing assistants were invited to participate. The providers were again asked to participate in a follow-up survey 4 months after the rollout $(n=41)$. A brief, 7 -item survey, including multiple-choice and free-response questions, was developed to assess the provider's use of CAIR, perceived impact on their workflow, and satisfaction with the hospital's immunization recording systems (Appendix). R for Mac (The R Foundation for Statistical Computing, Vienna, Austria) was used to perform Fisher's exact test for categorical variables, and the Wilcoxon Rank Sum test to compare the distribution of satisfaction ratings.

This project and study were done as part of quality improvement measures and thus IRB approval was not required.

\section{Results}

Figure 3 exemplifies that use of this tool has increased incrementally during the first 4 months following implementation. Data from LPCH was uploaded to the CAIR database starting in December 2011 - sharing all previously ordered immunizations and any immunizations ordered 
using CPOE after that date. Over 20,000 new immunization records - both historic and recentwere successfully inserted into the CAIR database since initiation.

Forty-one independent providers responded to each of the pre- and post-surveys as seen in Table 1. There was a slight statistical difference of the demographic distribution, as more attending physicians participated in the post-survey. Responders indicated increased usage of the registry following interface implementation $(\mathrm{p}=<0.001)$.

Of the providers surveyed, the majority had never accessed CAIR prior to the availability of the link. Afterwards, the number of providers who had accessed CAIR significantly increased ( $\mathrm{p}=<$ 0.001). Although, the majority of our respondents were physicians, the primary care clinic nursing staff reported the most frequent use of the tool.

Overall provider satisfaction increased slightly $(\mathrm{p}=0.009)$, however, the majority of providers $(56 \%)$ perceived that the smart link improved their efficiency (Figure 4). 68\% of the surveyed providers felt that the smart link increased the likelihood that their patients' immunization record was up to date (Figure 5).

Table 1. Respondents of pre- and post-intervention surveys

\begin{tabular}{|l|c|c|c|}
\hline & Pre-intervention & Post-intervention & P-value \\
\hline Number of respondents & 41 & 41 & \\
Position & & & $\mathrm{p}=0.04$ \\
Resident (\%) & $34(83)$ & $24(59)$ & \\
General Pediatrician (\%) & $3(7)$ & $11(27)$ & \\
Nurse (\%) & $4(10)$ & $5(12)$ & \\
Other (\%) & 0 & $1(2)$ & \\
& & & \\
Have you ever accessed CAIR? & & $21(51)$ & \\
Yes (\%) & $6(15)$ & $20(49)$ & \\
No (\%) & $33(80)$ & 0 & \\
Unsure $\%)$ & $2(5)$ & & \\
\hline
\end{tabular}




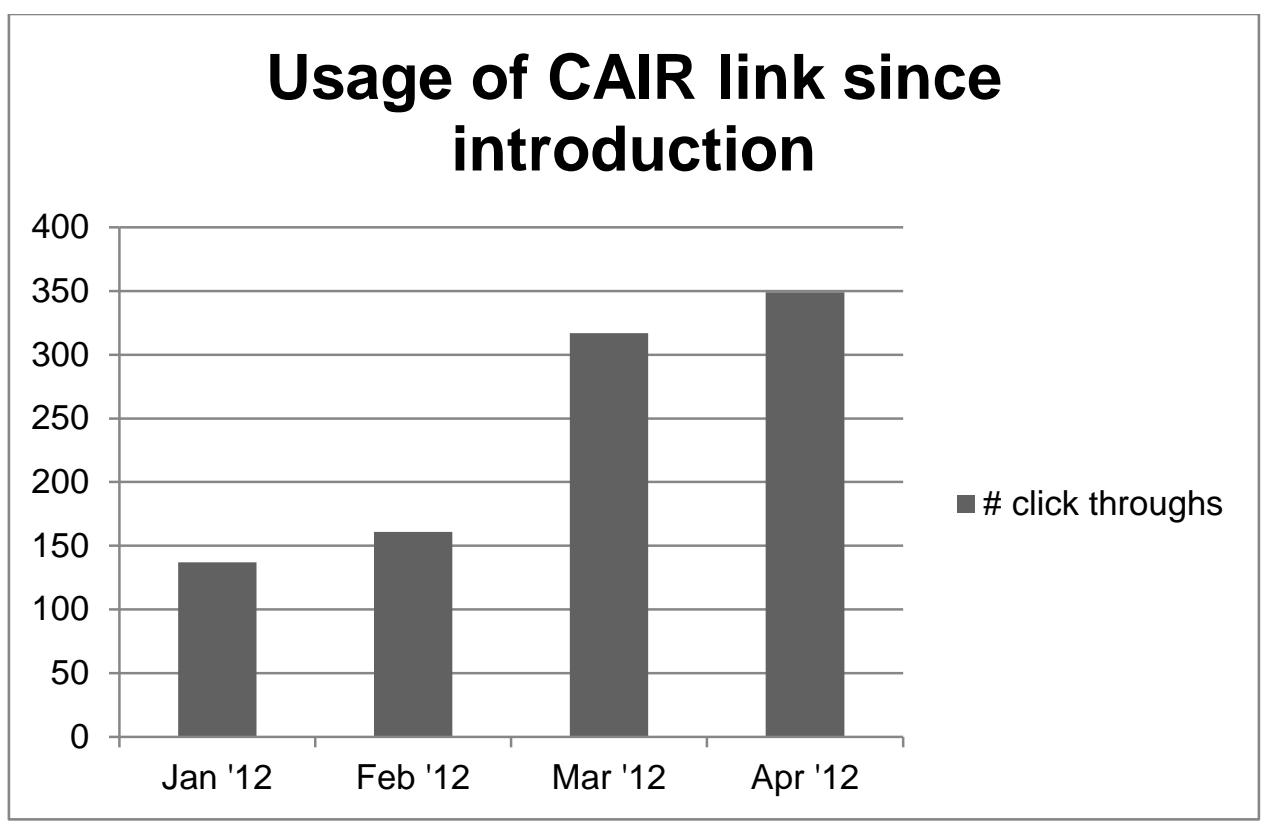

Figure 3: Usage of CAIR link since introduction

\section{The new EMR interface makes it more likely that my patients' immunization information is up to date.}

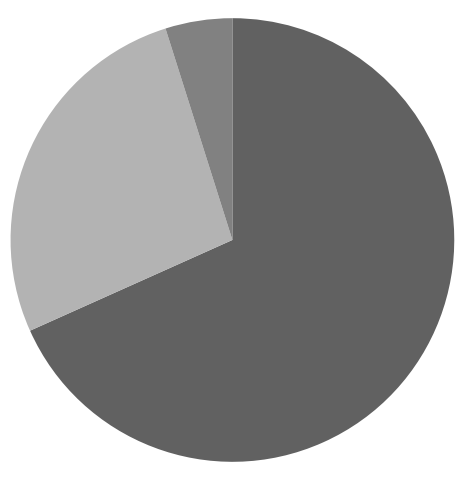

- Agree or Strongly Agree

$\square$ Neutral

Disagree or Strongly Disagree

Figures 4: Post-intervention survey responses 


\section{Having my patients' immunization information accessible through the smart link has improved my efficiency}

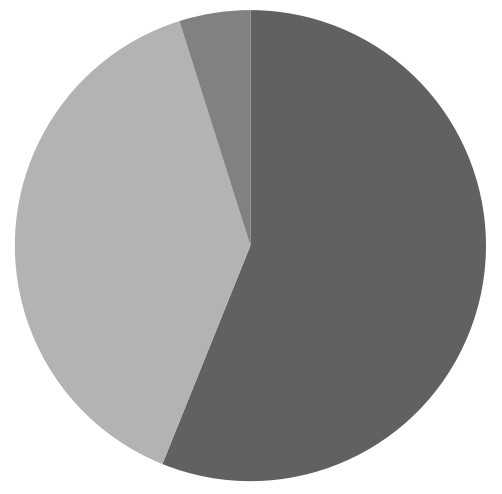

- Agree or Strongly Agree

Neutral

n Disagree or Strongly Disagree

Figures 5: Post-intervention survey responses.

\section{Discussion}

Few published examples of bidirectional interfaces between EMRs and immunization registries exist, and to the authors' knowledge, none about visual integration within a vendor EMR. ${ }^{17,}{ }^{18}$ The HITECH act lays out objectives in order to promote EMRs and their meaningful use in 3 successive stages. In order to receive economic incentives, each stage of criteria must be met, including those related to electronic data sharing with IIS. ${ }^{5,} 6,11,19$ While unidirectional interoperability is all that is required under $\mathrm{HITECH}$, visual integration of the registry is a significant enhancement because institutional immunization data is not only shared with the registry, individual patient data is easily accessible by providers within their workflow. ${ }^{11}$

These data suggest that use of CAIR among providers at LPCH has increased since the introduction of the interface to the EMR and a significant increase in the link utilization in the months since implementation is shown. An IIS is only as robust as the information it receives and the amount it is utilized. ${ }^{20}$ The CDC hopes to have $>95 \%$ of children under 6 included in an IIS by the year $2020,{ }^{21}$ a goal that will require participation of many additional institutions. Given the parallel federal incentives to implement EMR systems, many institutions are similarly hesitant to participate in an IIS due to concerns about extra work for staff. ${ }^{22,23}$

The interface implemented at LPCH is a web-based link, which preserves the sanctity of the registry database while improving provider workflow. System maintenance is relatively minimal once the algorithms are established. This link was established in a vendor-based system, which could be utilized by many other institutions. The only previously reported of bidirectional interface in NYC was with two smaller EMRs, both internally developed. ${ }^{17,}{ }^{18}$ Providers at LPCH indicated that having the link increased the likelihood that their patients' immunization information was up to date, as well as their overall efficiency. 
Although this study has a relatively small sample size, the authors feel that the responses reflect the general consensus of primary care providers at LPCH. Despite the slight difference in the demographics of the pre- and post- survey groups, it is unlikely that these differences bias the main outcome measures of this study. Another limitation is that the outpatient clinics at LPCH have not yet started utilizing CPOE to order immunizations, so the data shared with the IIS is currently limited to hospitalized inpatients, including newborns in the well baby nursery and obstetric patients. However, given that the link allows access to the entire registry's data set and not just patients who have had immunizations documented at $\mathrm{LPCH}$, its integrated nature makes it useful for providers (both inpatient and outpatient), who are able to examine records from patients' seen at other area clinics.

\section{Conclusions}

The successful implementation of a unidirectional interface between a commercial EMR and the California state immunization registry with visually integrated universal access has resulted in significant enhancement in the comprehensive nature of patient immunization records at this hospital.

It is the authors' hope that this example will serve to inspire other institutions with vendor-based EMR systems to implement similar interfaces for the good of all communities and patients.

\section{Acknowledgements}

The authors would like to acknowledge Joshua Faulkenberry, Pragati Kamath, and Nestor Llerena at LPCH, as well as Eric Dansby, Sarah Kang, and Jagadesh Talluri at CAIR, for their technical expertise and assistance with this project. Christopher Stave's assistance with the literature search is also greatly appreciated, as is Lisa Chamberlain's inspiration and advocacy leadership.

\section{Funding: None}

Financial Disclosure: The authors have no financial relationships relevant to this article to disclose.

Conflict of Interest: The authors have no conflicts of interest relevant to this article to disclose.

\section{Author Contribution Statements:}

Dr. Stevens helped conceptualize the project, administered the surveys, analyzed the data, drafted the manuscript, and approved the final version for submission.

Dr. Palma contributed to the statistical analysis, reviewed the manuscript, and approved the submission version.

Ms. Pandher helped design and worked with the technical development of the system, as well as aided in data acquisition and final approval of the manuscript. 
Dr. Longhurst contributed substantially to the conception, design, acquisition of data, analysis, and interpretation of data; revising the article for important intellectual content; and final approval of the published version.

\section{Corresponding Author}

Lindsay Stevens, MD

Department of Pediatrics

770 Welch Road, Suite 100

Palo Alto, CA 94304

lindsay.stevens@stanford.edu

650-497-8000

\section{References}

[1] Abramson J. S., O'Shea T. M., Ratledge D. L., Lawless M. R., Givner L. B. Development of a vaccine tracking system to improve the rate of age-appropriate primary immunization in children of lower socioeconomic status. J Pediatr. 1995;126(4):583-586.

[2] Linkins R. W. Immunization registries: progress and challenges in reaching the 2010 national objective. J Public Health Manag Pract. 2001;7(6):67-74.

[3] Placzek H., Madoff L. C. The use of immunization registry-based data in vaccine effectiveness studies. Vaccine. 2011;29(3):399-411.

[4] Weinberg ST. Immunization Registries: Where we've been and where we're headed. $A A P$ News. 2010;31(12):28.

[5] Meaningful Use and Immunization Information Systems. In.

[6] Meaningful use of electronic health records EHRs. In: American Academy of Pediatrics.

[7] Mahon B. E., Shea K. M., Dougherty N. N., Loughlin A. M. Implications for registry-based vaccine effectiveness studies from an evaluation of an immunization registry: a crosssectional study. BMC Public Health. 2008;8(160):160.

[8] Hinman A. R., Ross D. A. Immunization registries can be building blocks for national health information systems. Health Aff (Millwood).29(4):676-682.

[9] Centers for Disease Control and Prevention Immunization Registry Support Branch, National Immunization Program, Centers for Disease Control and Prevention. National Immunization Program. Implementation guide for immunization data transactions using version 2.3.1 of the Health Level Seven (HL7) Standard Protocol, version 2.2. In; 2006.

[10] Spooner S. A. Special requirements of electronic health record systems in pediatrics. Pediatrics. 2007;119(3):631-637.

[11] Dombkowski K. J., Clark S. J. Redefining meaningful use: achieving interoperability with immunization registries. Am J Prev Med. 2012;42(4):e33-35.

[12] Palma JP VanEaton EG, Longhurst CA. Neonatal Informatics: Information Technology to Support Handoffs in Neonatal Care. NeoReviews. 2011;12(10):e560-e563.

[13] Bernstein J Imler D, Sharek P, Longhurst C. Improved Physician Workflow and Satisfaction after Integration of Sign-out Notes into the EMR. Joint Commission Journal of Quality and Patient Safety. 2010.

[14] Frankovich J Longhurst CA, Sutherland SM. Evidence-based Medicine in the EMR Era. $N$ Engl J Med. 2011;365(19):1758-1759. 
[15] Longhurst CA Parast L, Sandborg CI, Widen E, Sullivan J, Hahn JS, Dawes CG, Sharek PJ. Decrease in Hospital-wide Mortality Associated with Implementation of a Comprehensive Electronic Medical Record. Pediatrics. 2010;126(1):14-21.

[16] Adams ES Longhurst CA, Pageler N, Widen E, Franzon D, Cornfield DN. Computerized physician order entry with decision support decreases blood transfusions in hospitalized children. Pediatrics. 2011;127(5):1112-1119.

[17] Arzt NH. Service-Oriented Architecture in Public Health: Interoperability Case Studies. Journal of Healthcare Information Management. 2010;24(2):45-52.

[18] Arzt NH Forney K, Chi A, Suralik M, Schaeffer P, Aponte A. Meaningful Use and Public Health: An Immunization Information System Case Study. Journal of Healthcare Information Management. 2011;25(4):37-44.

[19] Blumenthal D. Launching HITECH. N Engl J Med. 2009;362(5):382-385.

[20] Davidson A. J., Melinkovich P., Beaty B. L., Chandramouli V., Hambidge S. J., Phibbs S. L., et al. Immunization registry accuracy: improvement with progressive clinical application. Am J Prev Med. 2003;24(3):276-280.

[21] Progress in immunization information systems - United States, 2010. MMWR Morb Mortal Wkly Rep. 2012;61(25):464-467.

[22] Kairys S. W., Gubernick R. S., Millican A., Adams W. G. Using a registry to improve immunization delivery. Pediatr Ann. 2006;35(7):500-506.

[23] Saville A. W., Albright K., Nowels C., Barnard J., Daley M. F., Stokley S., et al. Getting under the hood: exploring issues that affect provider-based recall using an immunization information system. Acad Pediatr. 2011;11(1):44-49. 


\section{Appendix - Survey questions}

\section{Pre-Survey}

1. What is your current position?

2. How often do you need to access immunization information about a patient?

3. Have you ever accessed the California Immunization Registry?

The questions below used the Likert scale Strongly Disagree to Strongly Agree (1-5):

4. I am satisfied with the current system of recording immunizations.

5. I feel that integrating the centralized immunization record into our electronic medical record will improve my workflow.

6. Using a centralized resource for immunization records will improve patient care.

7. Do you have any additional comments?

\section{Post-Survey}

1. What is your current position?

2. How often do you need to access immunization information about a patient?

3. Did you ever access the California Immunization Registry before the link in Cerner was available?

4. Have you ever accessed the California Immunization Registry using the link in Cerner?

5 . If so, how many times have you accessed it in the last 2 weeks?

6. How strongly do you agree or disagree with the following statements?

- I am satisfied with the current system of recording immunizations.

- Having my patients' immunization information accessible through the smart link has improved my efficiency.

- The new EMR interface makes it more likely that my patients' immunization information is up to date.

7. Do you have any additional comments? 